\title{
CHARACTERIZING SYSTEMATIC ERRORS IN THE FARADAY ROTATION RETRIEVAL FROM SMOS MEASUREMENTS
}

\author{
R. Rubino ${ }^{(1,3)}$, N. Duffo ${ }^{(1,3)}$, V. González-Gambau ${ }^{(2,3)}, F$. Torres $^{(1,3)}$, I. Corbella $^{(1,3)}$ and M. Martín-Neira ${ }^{(4)}$ \\ (1)Remote Sensing Laboratory, Universitat Politècnica de Catalunya, Barcelona, Spain \\ (2)Institute of Marine Sciences, CSIC, Barcelona, Spain \\ (3)Barcelona Expert Centre, Barcelona, Spain \\ (4)European Space Agency, ESA-ESTEC, Noordwijk, The Netherlands
}

\begin{abstract}
In this work, a methodology to correct the Faraday Rotation Angle (FRA) is presented. It consists of calculating a systematic error pattern introduced by MIRAS. This error is calculated as follows: an area is chosen where it can be assumed that the FRA is very small. Therefore, the measure in this area is supposed to be a systematic error of the instrument. Once calculated, this error is subtracted in the rest of the measurements. In both cases, the FRA is calculated following a process of minimization of the equation that relates the SMOS full polarization radiometric measurements to that parameter.
\end{abstract}

Index Terms- Faraday rotation, Vertical Electron Content, L-band radiometry, interferometry, Soil Moisture and Ocean Salinity (SMOS)

\section{INTRODUCTION}

After 10 years of operation, the ESA's SMOS (Soil Moisture and Ocean Salinity) unique payload MIRAS (Microwave Imaging Radiometer using Aperture Synthesis) keeps providing good quality full polarimetric Brightness Temperature (TB) to generate frequent and global maps of the Earth's soil moisture and ocean salinity [1].

When crossing the ionosphere, the radiometric measurements rotate a certain angle caused by the Faraday effect. This effect is not negligible at SMOS operating frequency $(1.413 \mathrm{GHz})$ and must be compensated. This correction is done by applying equation (1) [3] [4]:

$$
\Omega_{f}=1.355 \times 10^{4} f^{-2} B_{0} \cos \Theta_{B} \sec \theta V T E C
$$

where $\Omega_{f}$ represents the Faraday rotation angle (FRA); $f$, the frequency in $\mathrm{GHz} ; \mathrm{B}_{0}$, the geomagnetic field in Tesla; $\Theta_{\mathrm{B}}$, the angle between the magnetic field and the wave propagation direction; $\theta$, the angle between the wave propagation direction and the vertical to the surface; and the Vertical Total Electron Content (VTEC) in TEC units $\left(10^{16}\right.$ electrons $\left./ \mathrm{m}^{2}\right)$. Both the geomagnetic field and the VTEC are given at a geodetic altitude of $400 \mathrm{~km}$. The geomagnetic field corresponds to a database that comes from International Geomagnetic Reference Field (IGRF) [4]. The Vertical Electron Content is read from a SMOS auxiliary data field called "consolidated TEC" (hereafter database VTEC [5]) for land and ascending orbits over the ocean. In the case of measurements over the ocean in descending orbits, VTEC values used to correct the FRA have been computed from the SMOS TB measurements following the methodology detailed in [6] and can be found in the OSDAP2 product [7].

Retrieving the FRA directly from the SMOS TB in a continuous way allows to improve the accuracy of the SMOS radiometric data. This is possible thanks to the latest advances in image reconstruction, which provide improved third and fourth Stokes parameters [8]. The FRA can be retrieved instantaneously in the entire SMOS Extended Alias FreeField of View (EAF-FoV) using radiometric data by solving the expression in (2) (equivalent to equation (22) in [3]):

$$
\Omega_{f}=-\varphi-\frac{1}{2} \arctan \left(\frac{2 \Re e\left(T_{B}^{x y}\right)}{T_{B}^{x x}-T_{B}^{y y}}\right)
$$

where $\varphi$ is the geometrical rotation angle, which is specific to the platform attitude and instrument orientation, and the terms $T_{B}^{x x}, T_{B}^{y y}, T_{B}^{x y}$ correspond to SMOS full-pol TB.

In order to mitigate the radiometric errors (calibration inaccuracies, image reconstruction artifacts, and antenna pattern uncertainties) when retrieving FRA in a single snapshot, the application of spatio-temporal filtering techniques is required. A recently developed methodology has allowed to derive VTEC maps from SMOS radiometric data over the AF-FoV to compensate the Faraday rotation [9].

In this work, a new methodology is proposed. It is focused on computing the systematic error pattern found in zones where the FRA should tend to zero. First, we have characterized this FRA systematic error and checked that it is time-independent. This contribution can then be used in the FRA calculation everywhere else. Finally, VTEC maps are derived to be used in the correction of the Faraday rotation effect in SMOS.

\section{METHODOLOGY}

MIRAS provides a block of full polarimetric TBs every 2.4 s. These TBs are used in equation (2) to compute the FRA in an instantaneous way. The SMOS brightness temperatures used in this work have been processed by the MIRAS Testing Software (MTS), developed by the Polytechnic University of Catalonia (UPC) [10].

The Faraday rotation can also be computed by minimizing the following expression derived from equation (2): 


$$
\left(T_{B}^{x x}-T_{B}^{y y}\right) * \tan \left(2\left(\Omega_{f}+\varphi\right)\right)-2 \Re\left(T_{B}^{x y}\right)=0
$$

It was found in [9] that in zones where the $\Omega_{f}^{m}$ (measured FRA) should tend to 0 , a systematic error pattern was affecting the FRA retrieval $\left(\Omega_{f}^{m}=\Omega_{f}+\Delta\right)$.

The proposed methodology works in three steps. First, the systematic error, called from now on delta $(\Delta)$ snapshot, is computed. For doing that, we minimize the expression in (3) for those measurements where the FRA tends to 0 , that is $\Omega_{f}^{m}=\Delta$. In this way, we can retrieve the value of delta in the antenna reference frame $(\xi-\eta$ coordinates). SMOS data acquired in ascending orbits in periods and regions with the lowest values of FRA are used to calculate delta. Three days of data over the Pacific Ocean have been used. TBs measured in all the snapshots in the latitude range $\left[35^{\circ} \mathrm{S} 5^{\circ} \mathrm{S}\right]$ have been averaged as well as the geometrical rotation angle $\varphi$ to then, obtain the FRA by minimizing equation (3). An indetermination emerges when $2\left(\Omega_{f}^{m}+\varphi\right) \approx \frac{\pi}{2}$ so a threshold is used to reject those pixels. When $T_{B}^{x x} \approx T_{B}^{y y}$, the expression is multiplied by zero making impossible the minimization. This happens at low incidence angles, which correspond to the bottom part of the snapshot. Only TBs measured at incidence angles higher than $27.5^{\circ}$ have been used.

The second step in the methodology consists of retrieving the FRA per snapshot in other regions where the values of FRA do not tend to 0 by minimizing equation (3) to obtain the values of the $\Omega_{f}^{m}$. Then, corrected FRA $\left(\Omega_{f}\right)$ values can be obtained by subtracting the delta contribution to the measured FRA $\left(\Omega_{f}^{m}\right)$.

In the third step, VTEC maps are derived from these FRA retrievals in order to dynamically correct for the FRA by using equation (1). The spatio-temporal filtering techniques used for retrieving VTEC maps are the same detailed in [9].

\section{PRELIMINARY RESULTS}

Figure 1 shows the latitude-time Hovmöller plot of the boresight ascending orbits FRA for the entire mission over the Pacific Ocean. From this figure, the periods with lower FRA values correspond to the last two years of the mission. This is caused by the low sun activity of those years and the relationship that there is between it and the VTEC. Figure 2 shows the systematic error pattern calculated with ascending orbits over the Pacific Ocean for two different periods (December $16^{\text {th }}$ to $18^{\text {th }}$ from 2017 (left) and 2018 (right)). Both patterns are very similar, which confirms that this systematic error is time-independent and can be computed once.

Using that delta snapshot, the VTEC from a descending orbit over the Pacific Ocean from March $21^{\text {st }} 2011$ has been retrieved (Figure 3.middle). This VTEC map can be compared with the database VTEC (Figure 3.top). The difference between the retrieved VTEC and the database is shown in Figure 3.bottom. The highest error is found in the Arctic Ocean close to Canada, which is caused by the presence of a Radio Frequency Interference (RFI) affecting SMOS TB measurements in that period [11]. Nonetheless, the retrieval follows the geophysical variation of the database VTEC even though it is generally lower, similarly to the results found when retrieving VTEC from SMOS data by other methodologies [6] [9].

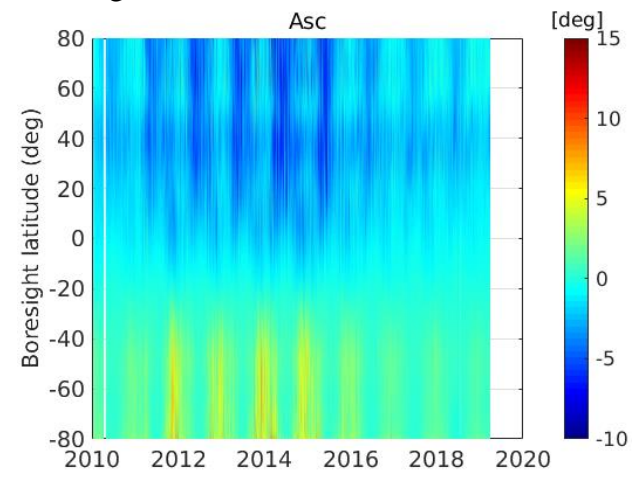

Figure 1. Latitude-time Hovmöller plot of the SMOS boresight ascending orbits FRA
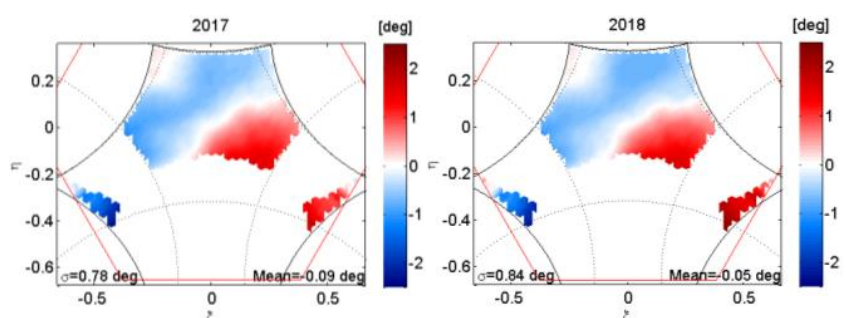

Figure 2. Systematic error pattern introduced by MIRAS for December: left: 2017, right: 2018.

The FRA can be computed from both VTEC datasets by applying equation (1). Figure 4 shows in red the FRA of a pixel in the middle of the swath with respect to latitude using the database VTEC and in green, using the retrieved VTEC. In Figure 5, the difference between both FRAs is shown. It can be seen that when processing SMOS radiometric data with the methodology, it is also possible to recover the FRA geophysical and temporal variation. The recovery is mostly lower than the database with an average difference of $-0.13^{\circ}$ degrees and a standard deviation of $0.85^{\circ}$. The gap in the recovery of both the VTEC (Figure 3) and the FRA (Figure 4 and 5) is due to the rejection of pixels causing the indetermination of equation (1), that is, when the geomagnetic field is orthogonal to the wave propagation direction. Between latitudes $55^{\circ} \mathrm{N}$ and $70^{\circ} \mathrm{N}$ is perceived how the retrieval is affected by the RFI. 

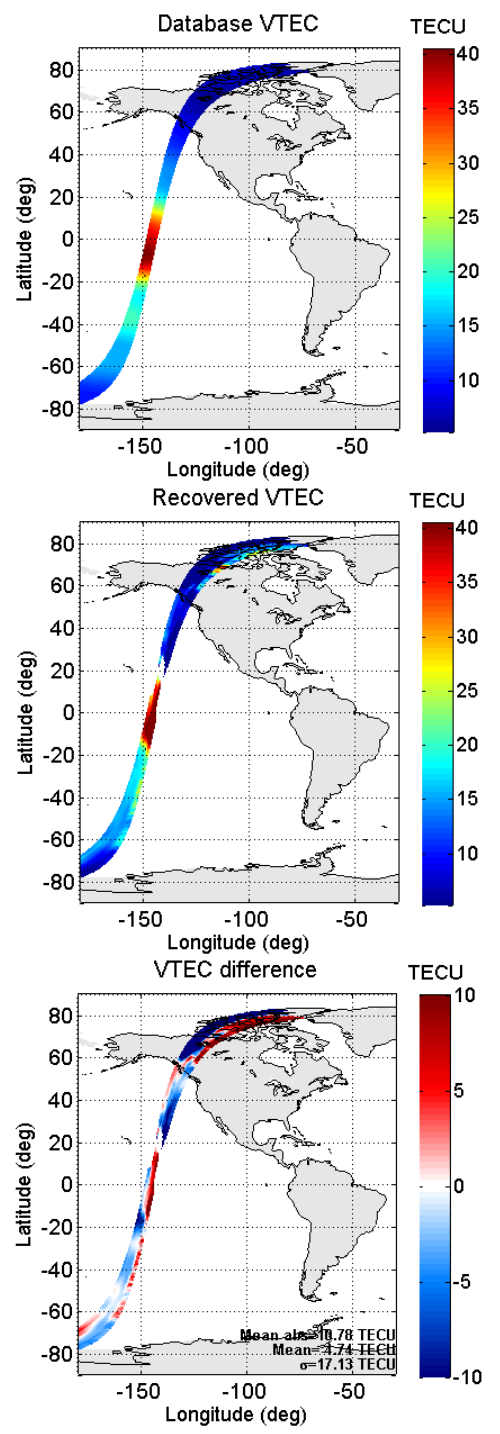

Figure 3. VTEC of a descendent orbit over the Pacific Ocean, March 21st, 2011. Top: value from the database, middle: retrieved VTEC from SMOS data with the proposed methodology, bottom: difference between the retrieval and the database VTEC.

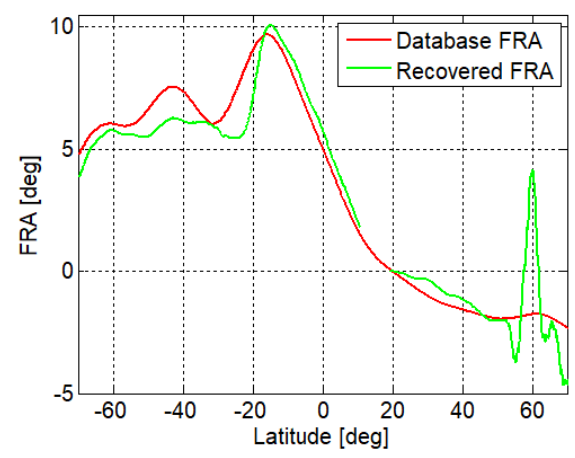

Figure 4. FRA of the middle pixel of the overpass vs lat of the database FRA (red) and retrieved FRA from SMOS data (green).

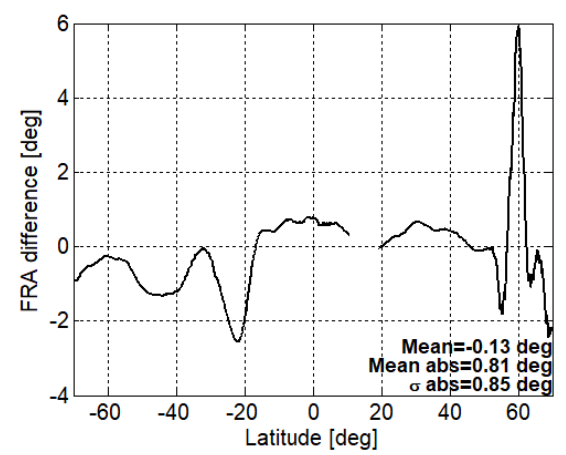

Figure 5. Difference between the recovered FRA from SMOS data and the database FRA of a middle pixel of the overpass vs lat.

\section{CONCLUSIONS}

The proposed improved methodology is able to retrieve VTEC maps from SMOS radiometric data in order to better correct the Faraday rotation effect. Preliminary results show that retrieved VTEC maps follow the geophysical variation shown in the database VTEC but with lower values, similarly to the results found when retrieving VTEC from SMOS data by other methodologies [6] [9]. Currently, global VTEC maps across different periods in time are being analyzed.

This methodology should work independently of the target seen by the instrument. However, we expect a lower performance of retrievals over land, since measurements can be affected by the presence of RFI sources. The analysis of the retrieval over land is currently on going.

Following steps will address the intercomparison of these results with other VTEC datasets: the VTEC in SMOS level 2 product (OSDAP2 [7]), the VTEC dataset coming from GPS data [12], and the VTEC maps retrieved by using the methodology described in [8]. Further work would be focused on evaluating the impact of using the different VTEC products in the computation of the Ocean Target Transformation used by the SMOS Ocean Salinity team to correct for spatial biases in TB [13]. This could be used as a metric of TB improvements prior to the salinity retrievals.

\section{ACKNOWLEDGMENT}

This work has been supported by the European Space Agency and Deimos Engenharia (Portugal), SMOS P7 Subcontract DME CP12 no. 2015-005; ERDF (European Regional Development Fund) and by Spanish public funds, projects TEC2017-88850-R and ESP2015-67549-C3-1-R; and through the award "Unidad de Excelencia María de Maeztu" MDM-2016-0600, financed by the "Agencia Estatal de Investigación" (Spain) and the European Regional Development Fund (ERDF).

\section{REFERENCES}

[1] M. Martín-Neira et al., "Polarimetric mode of MIRAS," 
IEEE Transactions on Geoscience and Remote Sensing, vol. 40, no. 8, pp. $1755-1768$, August 2002.

[2] D. M. Le Vine and S. Abraham, "The effect of the ionosphere on remote sensing of sea surface salinity from space: Absorption and emission at L band," IEEE Transactions on Geoscience and Remote Sensing, vol. 40, no. 4, pp. 771-782, April 2002.

[3] S. H. Yueh, "Estimates of Faraday rotation with passive Microwave polarimetry for microwave remote sensing of earth surfaces," IEEE Transactions on Geoscience and Remote Sensing, vol. 38, no. 5, pp. 2434-2438, September 2000.

[4] P. Alken, et al., "NOAA/NGDC candidate models for the $12^{\text {th }}$ Generation International Geomagnetic Reference Field," Earth Planets Space, vol. 67. 2015.

[5] J. Barbosa, "SMOS Level 1 and Auxiliary Data Products Specifications," Indra Systems, 2014.

[6] J.L. Vergely et al., "New total electron content retrieval improves SMOS sea surface salinity", J. Geophys. Res. Oceans, vol. 119, pp. 7295-7307, 2014, doi:10.1002/2014JC010150.

[7] B., Bengoa, "SMOS Level 2 and Auxiliary Data Products Specifications," 2017.

[8] L. Wu, et al., "Radiometric performance of SMOS full polarimetric imaging," IEEE Geoscience and Remote Sensing Letters, vol. 10, no. 6, pp. 1454-1458, November 2013.

[9] R. Rubino, N. Duffo, V. González-Gambau, I. Corbella, F. Torres, I. Durán, and M. Martín-Neira, "Deriving VTEC maps from SMOS radiometric data," submitted to Remote Sensing, January 2020.

[10] I. Corbella, F. Torres, N. Duffo, V. Gonzalez, A. Camps, M. Vall-llossera, "Fast Processing Tool for SMOS Data". In Proceedings of the IGARSS 2008 - 2008 IEEE International Geoscience and Remote Sensing Symposium; IEEE: Boston, MA, 2008; pp. II-1152 - II-1155.

[11] R. Oliva, et al., "Status of Radio Frequency Interference (RFI) in the 1400-1427 MHz passive band based on six years of SMOS mission," Remote Sensing Environment, 180, pp. 64-75. 2016.

[12] Hernandez-Pajares, M. (2003), Performances of IGS ionosphere TEC maps, 7th IGS Iono WG report, UPC, Barcelona, Spain.

[13] J. Tenerelli, N. Reul, "Analysis of L1PP calibration approach impacts in SMOS TB and 3-days SSS retrievals over the Pacific using an alternative Ocean Target Transformation applied to L1OP data," IFREMER/CLS, Tech. Rep., 2010. 